\title{
Ethic Statement Correction: Successful Endovascular Treatment of Cerebral Venous Thrombosis with a Novel, Larger Aspiration Catheter (REACT): A Case Report
}

Kaushik Sundar, DM, Sabharisundarvel Paulraj, MD, Shuvro Roy Choudhury, MD, Haseeb Hassan, DM, Judhajit Sengupta, MD, Sanjib Kumar Pattari, MD

Rabindranath Tagore International Institute of Cardiac Sciences, Kolkata, India

Correction to: Neurointervention https://doi.org/10.5469/neuroint.2020.00304, published on March, 2021, Neurointervention 2021;16:83-87.

In the originally published version of this article, there were some missing contents in the Ethics Statement section, which should be properly revised as follows:

\section{Before correction:}

\section{Ethics Statement}

Compliant with HIPAA guidelines.

\section{After correction:}

\section{Ethics Statement}

Compliant with HIPAA guidelines. Informed consent for publication was obtained from the patient and/or patient family.

The authors apologize for any inconvenience that it may have caused.

\section{Correspondence to: Kaushik Sundar, DM}

Rabindranath Tagore International Institute of Cardiac Sciences, 124, Mukundapur, EM Bypass, Kolkata 700099, West Bengal, India

Tel: +919585515234, E-mail: KAUSHIK.SUNDAR.DR@narayanahealth.org 